\title{
Factors and Outcome of Renal Osteodystrophy- Associated Initial Fragility Fracture in End-Stage Renal Disease Patients
}

\author{
Cai Li ${ }^{a} \quad$ Xue-mei Chen ${ }^{b} \quad$ Yin Li ${ }^{a, c}$ Yan-lin Zhou ${ }^{a}$ Jia-ni Yan ${ }^{a}$ Xiao-gang Du \\ a Department of Nephrology, The First Affiliated Hospital of Chongqing Medical University, Chongqing, China; \\ ${ }^{b}$ Emergency Department, The First Affiliated Hospital of Chongqing Medical University, Chongqing, China; \\ 'Department of Nephrology and Endocrinology, The People's Hospital of Tongliang District, Chongqing, China
}

\section{Keywords}

Renal osteodystrophy · Fragility fracture - Metastatic calcification - Bone and mineral metabolism · End-stage renal disease

\begin{abstract}
Background: Renal osteodystrophy has caused increased risk of fragility fracture in end-stage renal disease (ESRD) patients. However, risk factors and outcome of ESRD patients with fragility fracture remain uncharacterized. We aimed to assess these parameters in ESRD patients. Summary: This retrospective case-control study analyzed 354 ESRD patients (initial fragility fracture [FF] group, $n=59$; control group, $n=$ 295). Pre-dialysis blood hemoglobin, serum albumin, lipid, calcium, phosphorus, alkaline phosphatase (ALP), and intact parathyroid hormone (iPTH) were collected. All procedures performed involving human participants were in accordance with the ethical standards of the institutional committee of The First Affiliated Hospital of Chongqing Medical University (IRB approval number 216-82), and informed consent was obtained from all participants. There were higher prevalence rates of primary hypertension and diabetes, higher serum ALP, corrected calcium, and lower serum total cholesterol, low-density lipoprotein, lipoprotein-a, and iPTH in the
\end{abstract}

FF group. Fractures were more likely to occur in the higher level of corrected calcium as well as in the lower iPTH group. High corrected calcium $(p=0.010, \mathrm{OR}=11.308,95 \% \mathrm{Cl}$ : $1.770-72.242)$ and serum ALP $(p=0.000, \mathrm{OR}=1.007,95 \% \mathrm{Cl}$ : 1.004-1.011) were independent risk factors of fragility fracture. The incidence of all-cause mortality and cardiovascular (CV) events in ESRD patients with fragility fracture was higher than in those without fracture. Key Messages: Patients with hypertension, diabetes, excessive suppression of PTH, and poor nutritional status are more prone to fractures. Serum corrected calcium and ALP were independent risk factors of fragility fracture. Patients with initial fragility fracture had more CV events and higher mortality.

๑) 2019 S. Karger AG, Basel

\section{Introduction}

Renal osteodystrophy (ROD) is a common complication of chronic kidney disease (CKD), which may lead to defective mineralization, altered bone morphology, and/ or bone turnover [1-3]. Animal research found that bone

C.L. and X.C. contributed equally to this work and should be considered co-first authors.

\section{KARGER}

(c) 2019 S. Karger AG, Basel

E-Mail karger@karger.com

www.karger.com/kdd
Xiao-gang Du

Department of Nephrology

The First Affiliated Hospital of Chongqing Medical University

Youyi Road 1, Chongqing 400042 (China)

E-Mail dxgcxm@163.com 
changes occur even in the early stage of CKD [4], and with CKD progression, the patient may show symptoms such as bone pain, joint pain, bone deformation, and even spontaneous fractures.

CKD increases the risk of fractures [5], which are consequently associated with increased mortality, decreased quality of life, and higher economic burden. However, only few case reports $[6,7]$ and studies $[8,9]$ specifically focus on fractures in end-stage renal disease (ESRD) patients. In Chinese ESRD patients, fragility fractures remain uncharacterized, with the underlying mechanisms and risk factors undefined.

Recently, a preliminary epidemiologic investigation [8] showed that besides dialysis, old age, female gender, diabetes and liver cirrhosis, osteoporosis, and a prior history of hip fracture are also risk factors for hip fracture in patients with ESRD. Bone strength is mainly controlled by bone density, composition, and mineralization [10]. Alterations of bone turnover and defective mineralization are associated with increased risk of fracture. Recently, the organization of Kidney Disease: Improving Global Outcomes (KDIGO) proposed a new classification for ROD characterization, which encompasses changes in bone turnover, mineralization, and bone volume [11] However, the exact associations of bone turnover and bone metabolism with fracture incidence in Chinese ROD patients remain unstudied.

In this study, we assessed the clinical characteristics and risk factors of Chinese ESRD patients with initial fragility fracture, in order to prevent ROD-associated fracture and provide evidence for therapy improvement.

\section{Methods and Materials}

\section{Study Subjects}

We conducted a retrospective case-control study on hospitalized ESRD patients who received maintenance hemodialysis for at least 3 months at the First Affiliated Hospital of Chongqing Medical University from January 1, 2012 to September 30, 2016. Fiftynine cases were hospitalized for bone pain that occurred spontaneously or sustained from a minor trauma (a fall from standing height, sitting position, horizontal positioning, from 1-3 steps) and confirmed initial fragility fracture according to history of present illness and X-ray or CT examination, assigned to the fragility fracture group (FF group). A total of 295 hemodialysis patients without fracture hospitalized in the same period were selected as control group, matched in a 5:1 ratio for age, sex, and dialysis duration. The exclusion criteria were: incomplete data, high energy trauma fractures (motor vehicle accident, high-velocity bicycle accident, fall from significant height, etc.), old fractures, malignancy, tuberculosis, Cushing syndrome, current or past history of treatment with selective estrogen receptor modulators, and accepted glucocorticosteroid treatment for more than 2 years. Etiologies of the 354 enrolled ESRD patients are shown intuitively by a pie chart, and the respective etiologies of the two groups are shown by a chart in the online supplementary material 1 (for all online suppl. material, see www.karger.com/doi/10.1159/000494924). The 354 patients accepted the therapy of active vitamin D and calcic phosphorous binder from the stage of CKD3. A few of them may have changed the calcic agent to non-calcic phosphorous binder such as sevelamer or lanthanum carbonate. The usage of phosphorous binder within 3 months before enrollment is listed in the online supplementary material 2 . There was no difference between the two groups. All dialysate $\mathrm{Ca}$ concentrations were not higher than $1.25-1.50 \mathrm{mmol} / \mathrm{L}$.

\section{Data Collection}

Laboratory variables including blood hemoglobin, serum calcium, phosphorus, alkaline phosphatase (ALP), total cholesterol (TC), triglyceride (TG), low-density lipoprotein (LDL), highdensity lipoprotein (HDL), lipoprotein- $a(\mathrm{LP}-\alpha)$, prealbumin, albumin, and intact parathyroid hormone (iPTH) were collected before one regular dialysis at the time of enrollment. Calcium was corrected for serum albumin levels $<40 \mathrm{~g} / \mathrm{L}$ as follows: corrected calcium $(\mathrm{mmol} / \mathrm{L})=$ measured calcium $(\mathrm{mmol} / \mathrm{L})+0.2 \times(4-$ measured albumin [g/dL]) [12]. General information and body mass index (BMI) of the patients were recorded. Bone or cartilage structure fracture was defined as continuous bone interruption including epiphyseal separation, and evaluated by X-ray or CT. Radiological findings were analyzed by two observers blinded to clinical data, with an interobserver concordance of $95 \%$. Vascular calcification was determined with a CT value of vessel of 130 or higher by CT scan [13]. All subjects underwent echocardiography on a Hewlett-Packard Sonos 100 device equipped with a $2.25-\mathrm{MHz}$ probe to verify the presence of cardiac valvular calcification, if $\geq 1 \mathrm{~mm}$ strong echo occurred in aortic and mitral, tricuspid leaf, or disc ring as assessed by Doppler echocardiography [14].

\section{Statistical Analysis}

Statistical analysis was performed with the SPSS 22.0 statistical software. Numeric data were presented as mean \pm SD or median (interquartile range) and analyzed by Student's $t$ test or Wilcoxon test. Categorical variables were expressed as percentage and analyzed by $\chi^{2}$ test. Logistic regression analyses were performed to assess possible relationships while controlling for relevant measured correlates. $p<0.05$ was considered statistically significant.

\section{Results}

\section{The Common Site of Initial Fragility Fracture}

In this study, the most common fracture site was the hip (including 19 upper femur and 7 pubic bone fractures), followed by vertebrae (including 10 thoracic vertebral, 8 lumbar vertebral, and 1 case with both types). The other parts affected were radius, clavicle, patella, fibula, and scapula. Several fracture sites are shown in Figure 1 . 

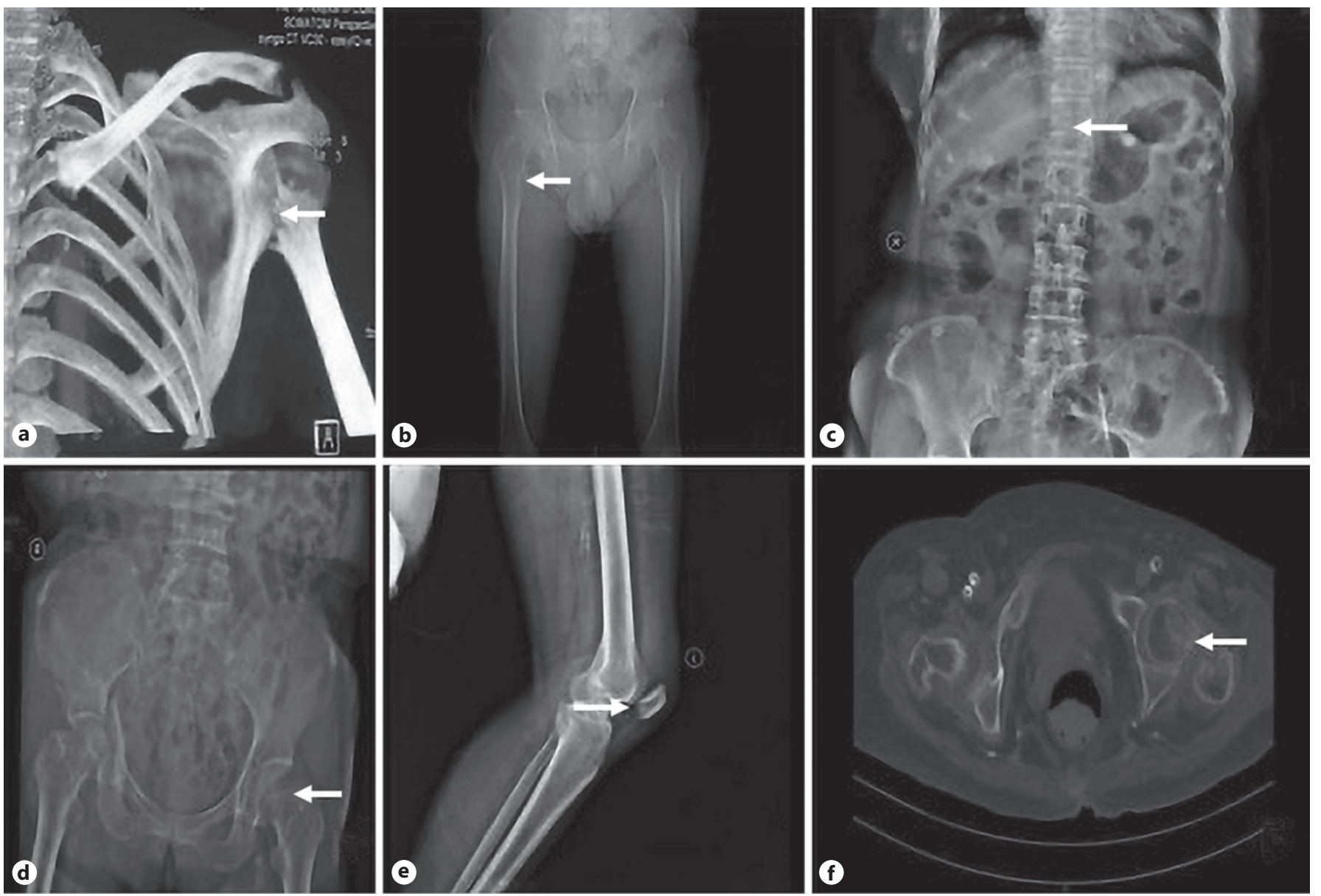

Fig. 1. Different fracture sites (arrows) revealed by X-ray or CT. a Fracture of left humerus head and surgical neck. b Avulsion fracture of right lesser trochanter. c Compression fracture of the twelfth thoracic spinal. d Left acetabular fracture. e Left patella fracture. $\mathbf{f}$ Left collum femoris fracture.

\section{Basic Clinical Characteristics of the Patients}

A total of 354 ESRD patients aged 20-90 years were enrolled in this study, including 182 males and $172 \mathrm{fe}$ males. There were 59 initial fragility fracture patients aged $65.3 \pm 14.3$ years in the FF group, including 26 males and 33 females, and 295 patients aged $61.7 \pm 14.2$ years in the control group, including 156 males and 139 females. Then, the primary causes of ESRD with or without fragility fracture were assessed, and higher prevalence rates of primary hypertension and diabetes mellitus were obtained in ESRD patients with initial fragility fracture compared with the control group $(p<$ 0.05 ). No significant differences in BMI and glucocorticosteroid treatment were found between the two groups (Table 1).

\section{Nutritional State of ESRD Patients with Initial}

\section{Fragility Fracture}

Nutritional state is often an important influential factor for fracture occurrence; therefore, we explored the correlation between the nutritional index and initial fragility fracture in ESRD patients. As shown in Table 1, there were lower serum TC, LDL, LP- $\alpha$ while higher hemoglobin levels $(p<0.05)$ in the FF group compared with control group values. No significant differences were obtained in serum albumin, prealbumin, TG, and HDL levels between the two groups.

\section{Mineral-Bone Metabolism Associated Parameters of}

ESRD Patients with Initial Fragility Fracture

Alterations of mineral-bone metabolism are associated with increased risk of fracture. In this study, we ana- 
Table 1. Clinical data, biochemical data, metastatic calcification, and outcomes in the two groups

\begin{tabular}{lccc}
\hline & $\begin{array}{c}\text { FF group } \\
(n=59)\end{array}$ & $\begin{array}{c}\text { Control group } \\
(n=295)\end{array}$ & $p$ value \\
\hline BMI & $22.89(19.48,25.08)$ & $22.66(20.18,24.71)$ & 0.984 \\
Glucocorticosteroid treatment, $n(\%)$ & $14(3.7)$ & $108(36.6)$ & 0.08 \\
Primary hypertension, $n$ \%) & $28(47.5)^{\mathrm{a}}$ & $89(30.2)$ & 0.042 \\
Diabetes, $n$ (\%) & $27(45.8)^{\mathrm{a}}$ & $95(32.2)$ & 0.045 \\
Hemoglobin, g/L & $102.0 \pm 24.14^{\mathrm{a}}$ & $93 \pm 25.0$ & 0.012 \\
Prealbumin, mg/L & $126.15 \pm 20.15$ & $129.14 \pm 20.06$ & 0.298 \\
Albumin, g/L & $36.3 \pm 5.90$ & $36.3 \pm 5.91$ & 0.968 \\
TC, mmol/L & $3.55(3.06,4.16)^{\mathrm{a}}$ & $4.02(3.34,4.75)$ & 0.019 \\
TG, mmol/L & $1.29(1.01,1.70)$ & $1.26(0.92,1.86)$ & 0.846 \\
LDL, mmol/L & $1.87(1.45,2.46)^{\mathrm{b}}$ & $2.21(1.70,2.96)$ & 0.007 \\
HDL, mmol/L & $1.13 \pm 0.35$ & $1.23 \pm 0.38$ & 0.084 \\
LP- $a$, mg/L & $234(106.0,445.0)^{\mathrm{a}}$ & $405(184.8,561.8)$ & 0.015 \\
ALP, U/L & $99.0(70.0,173.0)^{\mathrm{a}}$ & $86.0(67.0,114.0)$ & 0.014 \\
Calcium, mmol/L & $2.19(2.03,2.32)^{\mathrm{c}}$ & $2.10(1.87,2.20)$ & 0.000 \\
Corrected calcium, mmol/L & $2.27(2.15,2.42)^{\mathrm{b}}$ & $2.18(2.00,2.30)$ & 0.000 \\
Phosphorus, mmol/L & $1.60(1.24,1.81)$ & $1.76(1.37,1.98)$ & 0.060 \\
iPTH, pg/mL & $207.9(134.0,448.7)^{\mathrm{b}}$ & $362.5(222.4,503.5)$ & 0.002 \\
Calcium-phosphorus product, $\mathrm{mmol}^{2} / \mathrm{L}^{2}$ & $3.63(2.71,4.25)$ & $3.60(2.91,4.27)$ & 0.594 \\
Aortic calcification, $n(\%)$ & $32(54.2)^{\mathrm{b}}$ & $94(31.9)$ & 0.002 \\
Cardiac valve calcification, $n(\%)$ & $17(28.8)^{\mathrm{a}}$ & $43(14.6)$ & 0.013 \\
All-cause death, $n(\%)$ & $34(57.6)^{\mathrm{c}}$ & $51(17.3)$ & 0.000 \\
CV events, $n(\%)$ & $26(44.1)^{\mathrm{a}}$ & $85(28.8)$ & 0.017 \\
\hline
\end{tabular}

CV events include stable or unstable angina, myocardial infarction, heart failure, transient ischemic attack, cerebral ischemic stroke and subarachnoid hemorrhage, cerebral hemorrhage, peripheral arterial diseases, abdominal aortic aneurysm, arrhythmia, and sudden cardiac death. ${ }^{\mathrm{a}} p<0.05$ versus control group; ${ }^{\mathrm{b}} p<0.01$ versus control group; ${ }^{c} p<0.001$ versus control group.

Table 2. Risk factors of initial fragility fracture in ESRD patients analyzed by logistic regression

\begin{tabular}{lccc}
\hline Variables at baseline & Wald $z$ value & $p$ value & OR (95\% CI) \\
\hline Hemoglobin (g/L) & 7.891 & 0.005 & $1.024(1.007-1.041)$ \\
ALP (U/L) & 15.131 & 0.000 & $1.007(1.004-1.011)$ \\
Corrected calcium (mmol/L) & 6.572 & 0.010 & $11.308(1.770-72.242)$ \\
iPTH (pg/mL) & 4.722 & 0.030 & $0.999(0.998-1.000)$ \\
\hline
\end{tabular}

lyzed the changes of mineral-bone metabolism-associated parameters in ESRD patients with initial fragility fracture. As shown in Table 1, higher serum ALP, corrected calcium, and lower serum iPTH levels were found in the FF group compared with the control group $(p<0.05)$. No significant differences in phosphorus and calcium-phosphorus product were found between the two groups.

We further assessed the incidence of initial fragility fracture in patients with different levels of serum corrected calcium, phosphorus, and iPTH. According to the KDIGO recommendations regarding target corrected calcium (2.1-2.5 $\mathrm{mmol} / \mathrm{L})$ and phosphorus (1.13-1.78 $\mathrm{mmol} / \mathrm{L}$ ) or K/DOQI guideline recommendations about target $\mathrm{iPTH}$ (150-300 pg/mL) in CKD5 patients, the 354 patients were divided into three subgroups: lower, target, and higher level subgroups, respectively. As shown in Figure 2, fractures were more likely to occur in the higher level subgroups by corrected calcium levels as well as in the lower iPTH group $(p<0.05)$. However, no differences were found among the various serum phosphorus subgroups $(p>0.05)$. 
Fig. 2. Initial fragility fracture incidence in the three subgroups of serum corrected calcium (Ca) (a), phosphorus (P) (b), and iPTH (c). Corrected Ca subgroups, total comparison: $\chi^{2}=8.99, p<0.05, * p<0.05$ versus higher corrected $\mathrm{Ca}$ group. $\mathrm{P}$ subgroups, total comparison: $\chi^{2}=0.56, p>$ 0.05. iPTH subgroups, total comparison: $\chi^{2}=5.33, p<0.05,{ }^{\#} p<0.05$ versus higher iPTH group.

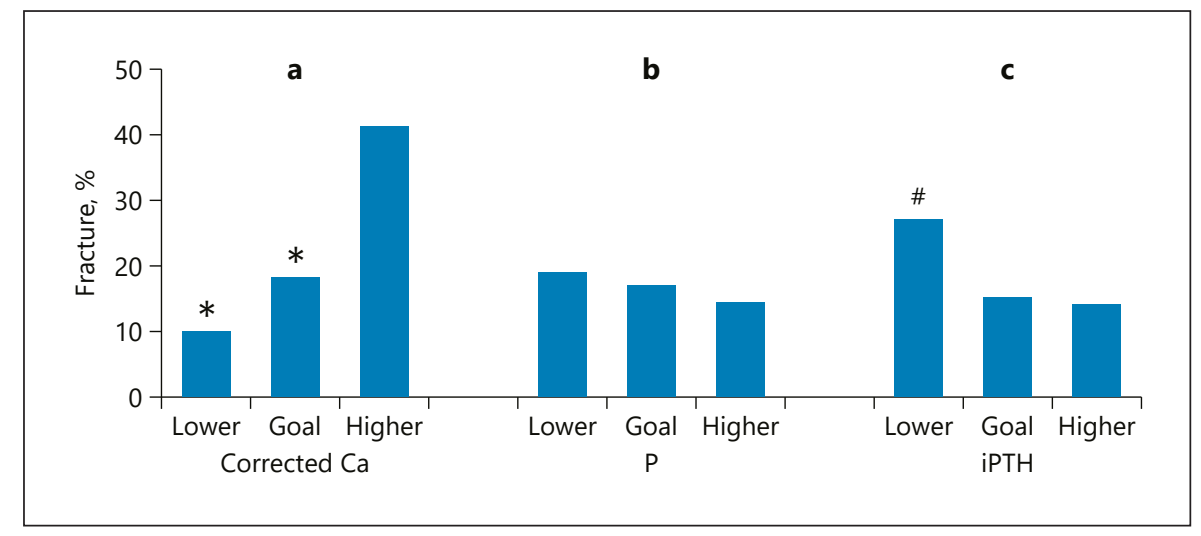

Risk Factors Associated with Initial Fragility Fracture Analyzed by Logistic Regression

All factors with $p<0.10$ in univariate analysis were included in a binary logistic regression model with backward selection. As shown in Table 2, higher serum corrected calcium and ALP were significant independent risk factors for initial fragility fracture in ESRD patients.

\section{Outcome Analyses}

We continued to follow-up all the investigated subjects till April 31, 2017, to collect the metastatic calcification (arterial and cardiac valve calcification), cardiovascular (CV) events, and all-cause death during the followup period. $1.7 \%$ patients were lost to follow-up halfway. 85 all-cause deaths were observed; 34 and 51 cases were in the FF and control groups, respectively. In the FF group, the ratios of aortic and cardiac valve calcification, $\mathrm{CV}$ events, and all-cause death were higher in the FF group compared with control group values (Table 1).

\section{Discussion}

\section{Hip Fractures Were the Most Common, Followed by}

\section{Vertebrae Fractures}

In this study, the hip (including femoral neck, greater trochanter, and pubic bone) was the most frequent fracture site, accounting for $44.1 \%$, followed by vertebrae fractures, which accounted for $32.2 \%$. Many studies [9, 11] showed that ESRD patients have significantly increased risk of fractures, with fragility fracture being a common complication of uremia. Based on US Renal Data System (USRDS) datasets, Wagner et al. [15] found that the incidence of vertebral and hip fractures in ESRD patients increased significantly, from $12.5 \%$ patient- years in 1992 to $25.3 \%$ patient-years in 2004 . Alem et al. [11] reported an annual incidence of hip fracture of $7.45 \%$ in male and $13.63 \%$ in female ESRD patients, respectively, i.e., 4.16 and 4.4 times higher than in normal gender-matched individuals. Lin et al. [8] reported an overall incidence rate of hip fracture of $89.21 \%$ patientyears for ESRD patients.

\section{Risk Factors of Fragility Fracture}

Fragility fracture is a common complication in patients with ESRD; however, the risk factors for fragility fracture remain undetermined. Firstly, we assessed the relationship between fragility fracture and the underlying primary disease of ESRD and found an increased risk of fragility fracture in ESRD patients with hypertension and diabetes; indeed, hypertension was independently associated with initial fragility fracture, corroborating previous reports [16-20]. A 10-year prospective study of 3,676 women in the United States showed that mineral (including urinary calcium) loss is increased in hypertension patients, which may constitute an important mechanism for fracture occurrence in hypertension patients with ESRD. Some scholars proposed that hypertension patients treated with calcium channel blockers show reduced bone absorption of calcium [21]. In addition, high blood pressure and blood pressure fluctuations increase the risk of falls and fracture [17]. Diabetes mellitus was found associated with increased fracture risk [22-25]. Blood sugar fluctuations may lead to increased risk of falls [26], and diabetic patients have bone fragility regardless of bone mineral density; the underlying mechanisms may include osteoblastic dysfunction, advanced glycation end products collagen cross-links, and micro-architectural abnormalities such as cortical porosity and deterioration of trabecular bone structure [27]. 
Poor nutritional status is a conditional risk factor for fracture. As shown above, serum TC, LDL, and LP- $\alpha$ levels were significantly lower in ESRD patients with initial fragility fracture. Sivas et al. [28] found that increasing TC by $1 \mathrm{mg} / \mathrm{dL}$ results in a $2.2 \%$ risk decrease for vertebral fractures. In agreement, Yamaguchi et al. [29] demonstrated that low TG levels are associated with vertebral fracture occurrence in postmenopausal women. Indeed, studies reported that osteoblasts and adipocytes originate from the same progenitor cells. It was demonstrated that the LDL receptor-related protein 5 (LRP5) gene plays an important role in bone mass loss and the risk of fractures [30]. In addition, drugs, such as statins and bisphosphonates, may affect bone and lipid metabolism [31, 32]. Besides, from clinical and nutritional standpoints, good nutritional status often means a good exercise of skeletal muscles, which prevents fracture in these patients. Unexpectedly, we found that higher hemoglobin levels in the FF group may be explained in this way: hyperhemoglobinemia often means increased blood viscosity, which results in endotheliocyte damage and micro-inflammatory state, thus affecting bone metabolism [33]. In fact, it was reported that hypohemoglobinemia and hyperhemoglobinemia are both associated with increased risk of fracture.

The pathogenesis of renal osteopathy is associated with an insufficient synthesis of $1,25(\mathrm{OH})_{2} \mathrm{D}$, hyperphosphatemia, hypocalcemia, and secondary hyperparathyroidism after renal failure [34, 35]. Interestingly, we found that serum corrected calcium levels were significantly higher in the FF group, and serum iPTH levels were lower compared with control group values; in addition, patients in the higher corrected calcium and lower PTH subgroups were more prone to fragility fracture, as shown by Figure 2, in agreement with previous findings [36]. Logistic regression analysis showed that fragility fracture was indeed associated with high corrected calcium levels. Similar studies have reported that hip fractures $[9,37,38]$ and vertebral body fractures [26] are starkly associated with low iPTH. In recent years, with a wide clinical use of calcium carbonate and vitamin D analogues, alongside improved dialysis techniques in ESRD, occurrence of high-bone turnover ROD has decreased, whereas hypercalcemia, excessive suppression of PTH, and secondary low-bone turnover ROD are increasingly prevalent and acknowledged [37, 39]. Although hyperphosphatemia, another important complication in ESRD, plays an important role in vascular calcification and affects prognosis of patients with ESRD [40], we found that hyperphosphatemia was not a risk factor for fracture in our study.

ROD-Associated Initial Fragility Fracture in ESRD Patients
As a representative of osteoblast activity, ALP plays an important role in bone calcification or tissue calcification. Maruyama et al. [41] demonstrated that elevated ALP is associated with reduced bone mineral density and may increase the risk of fracture in ESRD patients [42]. In this study, serum calcium and ALP levels in initial fragility fracture patients were significantly higher, but iPTH amounts were significantly reduced, with more severe arterial and cardiac valve calcification (Table 1). These findings suggested that elevated serum calcium cannot increase calcium deposits in the bone; on the contrary, it causes significantly increased metastatic calcification in soft tissues. An epidemiological study found that high serum ALP level may be associated with metastatic soft tissue calcification in ESRD patients [43]. Studies [44, 45] showed that elevated ALP amounts increase the hydrolysis of pyrophosphate, an inhibiting factor of vascular calcification, hence increasing the risk of metastatic calcification. Therefore, high serum ALP levels may also constitute a risk factor of initial fragility fracture and metastatic calcification in patients with ESRD.

\section{Outcome and Metastatic Calcification in ESRD \\ Patients}

ROD may influence the prognosis of ESRD patients. Mittalhenkle et al. [46] firstly focused on hip fracture and found that hip fracture was significantly correlated with high all-cause mortality in dialysis patients. In this study, we found that the CV events and all-cause death in the FF group were all higher than those in the control group.

To the best of our knowledge, this retrospective casecontrol study may be the first population-based investigation assessing the risk factors of fragility fracture in ESRD patients in China. Multiple reports showed that presence and seriousness of vascular calcification are associated with $\mathrm{CV}$ and all-cause mortality independently [39]. In this study, we found that ESRD patients with initial fragility fracture had more overt vascular calcification, heart valve calcification, and CV events, compared with the control group. Previous findings indicated that CKD-mineral and bone disorder, including metastatic calcification, in addition to causing significantly increased risk of fracture, is associated with higher CV mortality and morbidity. Indeed, in CKD, the bone may detrimentally impact vascular calcification through many ways, including altered secretion of $\mathrm{PTH}$, abnormal bone remodeling, calcium-phosphate disorders, and excessive vitamin D supplementation [47]. The present study revealed that Chinese ESRD patients with initial fragility fracture had higher incidence of metastatic calcification 
as well as increased risk of CV events and all-cause death compared with those without fracture.

This was a single-center study, with a sample size not large enough in the analysis, which may constitute a sampling error. The bone mineral density data were not taken into consideration in the analysis. A bone biopsy may offer a guide for fragility fracture prevention and treatment, but it was not carried out. The follow-up period was not long enough since some patients entered the study late, especially those enrolled in 2015-2016, which may introduce a bias into mineral-bone metabolism and outcome analyses, and if possible, we need to conduct a further survival analysis of ESRD patients.

In summary, this retrospective case-control study showed that hip fractures were the most common fracture site, serum corrected calcium and ALP were found to be independent risk factors for initial fragility fracture in patients with ESRD, and excessive suppression of PTH and poor nutritional status are unfavorable for ESRD patients. In addition, our findings demonstrated that ESRD patients with initial fragility fracture had more CV events and a poor outcome.

\section{Statement of Ethics}

The research was ethically conducted in accordance with the World Medical Association Declaration of Helsinki.

\section{Disclosure Statement}

The authors declare no conflicts of interest.

\section{References}

1 Gal-Moscovici A, Sprague SM. Bone health in chronic kidney disease-mineral and bone disease. Adv Chronic Kidney Dis. 2007 Jan; 14(1):27-36.

2 Elder G. Pathophysiology and recent advances in the management of renal osteodystrophy. J Bone Miner Res. 2002 Dec;17(12): 2094-105.

3 Kanjevac T, Bijelic B, Brajkovic D, Vasovic M, Stolic R. Impact of Chronic Kidney Disease Mineral and Bone Disorder on Jaw and $\mathrm{Al}$ veolar Bone Metabolism: A Narrative Review. Oral Health Prev Dent. 2018;16(1):79-85.

4 Bajwa NM, Sanchez CP, Lindsey RC, Watt H, Mohan S. Cortical and trabecular bone are equally affected in rats with renal failure and secondary hyperparathyroidism. BMC Nephrol. 2018 Feb;19(1):24.

5 Evenepoel P, Behets GJ, Laurent MR, D’Haese PC. Update on the role of bone biopsy in the management of patients with CKD-MBD. J Nephrol. 2017 Oct;30(5):645-52.

6 Garcia FL, Dalio RB, Sugo AT, Picado CH. Bilateral spontaneous fracturing of the femoral neck in a patient with renal osteodystrophy. Rev Bras Ortop. 2014 Jul;49(5):540-2.

7 Karapinar H, Ozdemir M, Akyol S, Ulkü O. Spontaneous bilateral femoral neck fractures in a young adult with chronic renal failure. Acta Orthop Belg. 2003;69(1):82-5.

8 Lin ZZ, Wang JJ, Chung CR, Huang PC, Su BA, Cheng KC, et al. Epidemiology and mortality of hip fracture among patients on dialysis: Taiwan National Cohort Study. Bone. 2014 Jul;64:235-9.

9 Al Helal B, Su WS, Churchill DN, Gangji AS. Relative hypoparathyroidism and hypoalbuminemia are associated with hip fracture in hemodialysis patients. Clin Nephrol. 2010 Feb;73(2):88-93.
10 Isaksson H, Turunen MJ, Rieppo L, Saarakkala S, Tamminen IS, Rieppo J, et al. Infrared spectroscopy indicates altered bone turnover and remodeling activity in renal osteodystrophy. J Bone Miner Res. 2010 Jun;25(6):13606.

11 Alem AM, Sherrard DJ, Gillen DL, Weiss NS, Beresford SA, Heckbert SR, et al. Increased risk of hip fracture among patients with endstage renal disease. Kidney Int. 2000 Jul;58(1): 396-9.

12 Klaus G, Watson A, Edefonti A, Fischbach M, Rönnholm K, Schaefer F, et al.; European Pediatric Dialysis Working Group (EPDWG). Prevention and treatment of renal osteodystrophy in children on chronic renal failure: european guidelines. Pediatr Nephrol. 2006 Feb;21(2):151-9.

13 Wilkieson TJ, Rahman MO, Gangji AS, Voss $\mathrm{M}$, Ingram AJ, Ranganath N, et al. Coronary artery calcification, cardiovascular events, and death: a prospective cohort study of incident patients on hemodialysis. Can J Kidney Health Dis. 2015 Aug;2:29.

14 Ellouali F, Berkchi F, Elhoussni S, Bayahia R, Benamar L, Abouqal R, et al. Evaluation of the effect of duration on dialysis on echocardiographic parameters: a preliminary study. Saudi J Kidney Dis Transpl. 2015 Jan;26(1):83-9.

15 Wagner J, Jhaveri KD, Rosen L, Sunday S, Mathew AT, Fishbane S. Increased bone fractures among elderly United States hemodialysis patients. Nephrol Dial Transplant. 2014 Jan;29(1):146-51.

16 Yang S, Nguyen ND, Center JR, Eisman JA, Nguyen TV. Association between hypertension and fragility fracture: a longitudinal study. Osteoporos Int. 2014 Jan;25(1):97103.
17 Sennerby U, Farahmand B, Ahlbom A, Ljunghall S, Michaëlsson K. Cardiovascular diseases and future risk of hip fracture in women. Osteoporos Int. 2007 Oct;18(10): 1355-62.

18 Vestergaard P, Rejnmark L, Mosekilde L. Hypertension is a risk factor for fractures. Calcif Tissue Int. 2009 Feb;84(2):103-11.

19 Maravic M, Ostertag A, Torres PU, CohenSolal M. Incidence and risk factors for hip frac-tures in dialysis patients. Osteoporos Int. 2014 Jan;25(1):159-65.

20 Wagner J, Jhaveri KD, Rosen L, Sunday S, Mathew AT, Fishbane S. Increased bone fractures among elderly United States hemodialysis patients. Nephrol Dial Transplant. 2014 Jan;29(1):146-51.

21 Fouquoire B, Poncelet P, Warembourg A, Petetin N, Debrueres S, Carre A. Calcium intestinal absorption in normotensive and essential hypertensive subjects before and after nicardipine. Arch Mal Coeur Vaiss. 1989;82(7): 1289-91.

22 Bonds DE, Larson JC, Schwartz AV, Strotmeyer ES, Robbins J, Rodriguez BL, et al. Risk of fracture in women with type 2 diabetes: the Women's Health Initiative Observational Study. J Clin Endocrinol Metab. 2006 Sep; 91(9):3404-10.

23 Schwartz AV, Vittinghoff E, Bauer DC, Hillier TA, Strotmeyer ES, Ensrud KE, et al.; Study of Osteoporotic Fractures (SOF) Research Group; Osteoporotic Fractures in Men (MrOS) Research Group; Health, Aging, and Body Composition (Health $\mathrm{ABC}$ ) Research Group. Association of BMD and FRAX score with risk of fracture in older adults with type 2 diabetes. JAMA. 2011 Jun; 305(21):2184-92. 
24 de Liefde II, van der Klift M, de Laet CE, van Daele PL, Hofman A, Pols HA. Bone mineral density and fracture risk in type-2 diabetes mellitus: the Rotterdam Study. Osteoporos Int. 2005 Dec; 16(12):1713-20.

25 Miao J, Brismar K, Nyrén O, UgarphMorawski A, Ye W. Elevated hip fracture risk in type 1 diabetic patients: a population-based cohort study in Sweden. Diabetes Care. 2005 Dec;28(12):2850-5.

26 Atsumi K, Kushida K, Yamazaki K, Shimizu $\mathrm{S}$, Ohmura A, Inoue T. Risk factors for vertebral fractures in renal osteodystrophy. Am J Kidney Dis. 1999 Feb;33(2):287-93.

27 Kanazawa I, Sugimoto T. [Diabetes mellitus and bone]. Clin Calcium. 2016 Aug;26(8): 1185-93.

28 Sivas F, Alemdaroğlu E, Elverici E, Kuluğ T, Ozoran K. Serum lipid profile: its relationship with osteoporotic vertebrae fractures and bone mineral density in Turkish postmenopausal women. Rheumatol Int. 2009 Jun; 29(8):885-90.

29 Yamaguchi T, Sugimoto T, Yano S, Yamauchi $\mathrm{M}$, Sowa $\mathrm{H}$, Chen Q, et al. Plasma lipids and osteoporosis in postmenopausal women. Endocr J. 2002 Apr;49(2):211-7.

30 van Meurs JB, Trikalinos TA, Ralston SH, Balcells S, Brandi ML, Brixen K, et al.; GENOMOS Study. Large-scale analysis of association between LRP5 and LRP6 variants and osteoporosis. JAMA. 2008 Mar;299(11): 1277-90.

31 Majima T, Komatsu Y, Fukao A, Ninomiya K, Matsumura T, Nakao K. Short-term effects of atorvastatin on bone turnover in male patients with hypercholesterolemia. Endocr J. 2007 Feb;54(1):145-51.
32 Herrington DM, Potvin Klein K. Statins, hormones, and women: benefits and drawbacks for atherosclerosis and osteoporosis. Curr Atheroscler Rep. 2001 Jan;3(1):35-42.

33 Looker AC. Hemoglobin and hip fracture risk in older non-Hispanic white adults. Osteoporos Int. 2014 Oct;25(10):2389-98.

34 Babayev R, Nickolas TL. Can one evaluate bone disease in chronic kidney disease without a biopsy? Curr Opin Nephrol Hypertens. 2014 Jul;23(4):431-7.

35 Drüeke TB. The pathogenesis of parathyroid gland hyperplasia in chronic renal failure. Kidney Int. 1995 Jul;48(1):259-72.

36 Iimori S, Mori Y, Akita W, Kuyama T, Takada $\mathrm{S}$, Asai T, et al. Diagnostic usefulness of bone mineral density and biochemical markers of bone turnover in predicting fracture in CKD stage 5D patients-a single-center cohort study. Nephrol Dial Transplant. 2012 Jan; 27(1):345-51.

37 Gal-Moscovici A, Sprague SM. Osteoporosis and chronic kidney disease. Semin Dial. 2007 Sep-Oct;20(5):423-30.

38 Coco M, Rush H. Increased incidence of hip fractures in dialysis patients with low serum parathyroid hormone. Am J Kidney Dis. 2000 Dec;36(6):1115-21.

39 London GM, Guérin AP, Marchais SJ, Métivier F, Pannier B, Adda H. Arterial media calcification in end-stage renal disease: impact on all-cause and cardiovascular mortality. Nephrol Dial Transplant. 2003 Sep;18(9): 1731-40.

40 Covic A, Rastogi A. Hyperphosphatemia in patients with ESRD: assessing the current evidence linking outcomes with treatment adherence. BMC Nephrol. 2013 Jul;14(1):153.
41 Maruyama Y, Taniguchi M, Kazama JJ, Yokoyama K, Hosoya T, Yokoo T, et al. A higher serum alkaline phosphatase is associated with the incidence of hip fracture and mortality among patients receiving hemodialysis in Japan. Nephrol Dial Transplant. 2014 Aug; 29(8):1532-8.

42 Park JC, Kovesdy CP, Duong U, Streja E, Rambod M, Nissenson AR, et al. Association of serum alkaline phosphatase and bone mineral density in maintenance hemodialysis patients. Hemodial Int. 2010 Apr;14(2):182-92.

43 Shantouf R, Kovesdy CP, Kim Y, Ahmadi N, Luna A, Luna C, et al. Association of serum alkaline phosphatase with coronary artery calcification in maintenance hemodialysis patients. Clin J Am Soc Nephrol. 2009 Jun;4(6): 1106-14.

44 Lomashvili KA, Garg P, Narisawa S, Millan JL, O’Neill WC. Upregulation of alkaline phosphatase and pyrophosphate hydrolysis: potential mechanism for uremic vascular calcification. Kidney Int. 2008 May;73(9):102430.

45 Schoppet M, Shanahan CM. Role for alkaline phosphatase as an inducer of vascular calcification in renal failure? Kidney Int. 2008 May; 73(9):989-91.

46 Mittalhenkle A, Gillen DL, Stehman-Breen CO. Increased risk of mortality associated with hip fracture in the dialysis population. Am J Kidney Dis. 2004 Oct;44(4):672-9.

47 Moe SM. Vascular calcification and renal osteodystrophy relationship in chronic kidney disease. Eur J Clin Invest. 2006 Aug;36(s2 Suppl 2):51-62. 